\title{
The Weakest Failure Detector for Eventual Consistency
}

\author{
Swan Dubois \\ Sorbonne Universités, UPMC \\ Univ Paris 06, CNRS, INRIA, \\ LIP6 UMR 7606
} Télécom ParisTech

\author{
Franck Petit \\ Sorbonne Universités, UPMC \\ Univ Paris 06, CNRS, INRIA, \\ LIP6 UMR 7606
}

\author{
Pierre Sens \\ Sorbonne Universités, UPMC \\ Univ Paris 06, CNRS, INRIA, \\ LIP6 UMR 7606
}

\begin{abstract}
In its classical form, a consistent replicated service requires all replicas to witness the same evolution of the service state. Assuming a message-passing environment with a majority of correct processes, the necessary and sufficient information about failures for implementing a general state machine replication scheme ensuring consistency is captured by the $\Omega$ failure detector.

This paper shows that in such a message-passing environment, $\Omega$ is also the weakest failure detector to implement an eventually consistent replicated service, where replicas are expected to agree on the evolution of the service state only after some (a priori unknown) time.

In fact, we show that $\Omega$ is the weakest to implement eventual consistency in any message-passing environment, i.e., under any assumption on when and where failures might occur. Ensuring (strong) consistency in any environment requires, in addition to $\Omega$, the quorum failure detector $\Sigma$. Our paper thus captures, for the first time, an exact computational difference between building a replicated state machine that ensures consistency and one that only ensures eventual consistency.
\end{abstract}

\section{Categories and Subject Descriptors}

C.2.4 [Computer-Communication Networks]: Distributed Systems; F.1.1 [Computation by Abstract Devices]: Models of Computation-relations between models

\section{Keywords}

eventual consistency, failure detectors

\section{INTRODUCTION}

State machine replication $[21,26]$ is the most studied technique to build a highly-available and consistent distributed

\footnotetext{
*The research leading to these results has received funding from the Agence Nationale de la Recherche, under grant agreement N ANR-14-CE35-0010-01, project DISCMAT.

Permission to make digital or hard copies of all or part of this work for personal or classroom use is granted without fee provided that copies are not made or distributed for profit or commercial advantage and that copies bear this notice and the full citation on the first page. Copyrights for components of this work owned by others than ACM must be honored. Abstracting with credit is permitted. To copy otherwise, or republish, to post on servers or to redistribute to lists, requires prior specific permission and/or a fee. Request permissions from permissions@acm.org. PODC'15, July 21-23, 2015, Donostia-San Sebastián, Spain. Copyright (C) 2015 ACM 978-1-4503-3617-8/15/07 ...\$15.00. DOI: http://dx.doi.org/10.1145/2767386.2767404.
}

service. Roughly speaking, the idea consists in replicating the service, modeled as a state machine, over several processes and ensuring that all replicas behave like one correct and available state machine, despite concurrent invocations of operations and failures of replicas. This is typically captured using the abstraction of a total order broadcast [3], where messages represent invocations of the service operations from clients to replicas (server processes). Assuming that the state machine is deterministic, delivering the invocations in the same total order ensures indeed that the replicas behave like a single state machine. Total order broadcast is, in turn, typically implemented by having the processes agree on which batch of messages to execute next, using the consensus abstraction $[22,3]$. (The two abstractions, consensus and total order broadcast, were shown to be equivalent [3].)

Replicas behaving like a single one is a property generally called consistency. The sole purpose of the abstractions underlying the state machine replication scheme, namely consensus and total order broadcast, is precisely to ensure this consistency, while providing at the same time availability, namely that the replicated service does not stop responding. The inherent costs of these abstractions are sometimes considered too high, both in terms of the necessary computability assumptions about the underlying system $[12,2$, $1]$, and the number of communication steps needed to deliver an invocation $[22,23]$.

An appealing approach to circumvent these costs is to trade consistency with what is sometimes called eventual consistency [25, 29]: namely to give up the requirement that the replicas always look the same, and replace it with the requirement that they only look the same eventually, i.e., after a finite but not a priori bounded period of time. Basically, eventual consistency says that the replicas can diverge for some period, as long as this period is finite.

Many systems claim to implement general state machines that ensure eventual consistency in message-passing systems, e.g., [20, 7]. But, to our knowledge, there has been no theoretical study of the exact assumptions on the information about failures underlying those implementations. This paper is the first to do so: using the formalism of failure detectors $[3,2]$, it addresses the question of the minimal information about failures needed to implement an eventually consistent replicated state machine.

It has been shown in [2] that, in a message-passing environment with a majority of correct processes, the weakest failure detector to implement consensus (and, thus, total order broadcast [5]) is the eventual leader failure detector, denoted $\Omega$. In short, $\Omega$ outputs, at every process, a leader 
process so that, eventually, the same correct process is considered leader by all. $\Omega$ can thus be viewed as the weakest failure detector to implement a generic replicated state machine ensuring consistency and availability in an environment with a majority of correct processes.

We show in this paper that, maybe surprisingly, the weakest failure detector to implement an eventually consistent replicated service in this environment (in fact, in any environment) is still $\Omega$. We prove our result via an interesting generalization of the celebrated "CHT proof" by Chandra, Hadzilacos and Toueg [2]. In the CHT proof, every process periodically extracts the identifier of a process that is expected to be correct (the leader) from the valencies of an ever-growing collection of locally simulated runs. We carefully adjust the notion of valency to apply this approach to the weaker abstraction of eventual consensus, which we show to be necessary and sufficient to implement eventual consistency.

Our result becomes less surprising if we realize that a correct majority prevents the system from being partitioned, and we know that both consistency and availability cannot be achieved while tolerating partitions $[1,14,8]$. Therefore, in a system with a correct majority of processes, there is no gain in weakening consistency: (strong) consistency requires the same information about failures as eventual one. In an arbitrary environment, however, i.e., under any assumptions on when and where failures may occur, the weakest failure detector for consistency is known to be $\Omega+\Sigma$, where $\Sigma$ [8] returns a set of processes (called a quorum) so that every two such quorums intersect at any time and there is a time after which all returned quorums contain only correct processes. We show in this paper that ensuring eventual consistency does not require $\Sigma$ : only $\Omega$ is needed, even if we do not assume a majority of correct processes. Therefore, $\Sigma$ represents the exact difference between consistency and eventual consistency. Our result thus theoretically backs up partition-tolerance $[1,14]$ as one of the main motivations behind the very notion of eventual consistency.

We establish our results through the following steps:

- We give precise definitions of the notions of eventual consensus and eventual total order broadcast. We show that the two abstractions are equivalent. These underlie the intuitive notion of eventual consistency implemented in many replicated services $[7,6,4]$.

- We show how to extend the celebrated CHT proof [2], initially establishing that $\Omega$ is necessary for solving consensus, to the context of eventual consensus. Through this extension, we indirectly highlight a hidden power of the technique proposed in [2] that somehow provides more than was used in the original $\mathrm{CHT}$ proof.

- We present an algorithm that uses $\Omega$ to implement, in any message-passing environment, an eventually consistent replicated service. The algorithm features three interesting properties: (1) An invocation can be performed after the optimal number of two communication steps, even if a majority of processes is not correct and even during periods when processes disagree on the leader, i.e., partition periods; ${ }^{1}$ (2) If $\Omega$ outputs the same leader at all processes from the very beginning, then the algorithm implements total order broadcast and hence ensures consistency; (3) Causal ordering is

\footnotetext{
${ }^{1}$ Note that three communication steps are, in the worst case,
} necessary when strong consistency is required [23]. ensured even during periods where $\Omega$ outputs different leaders at different processes.

The rest of the paper is organized as follows. We present our system model and basic definitions in Section 2. In Section 3, we introduce abstractions for implementing eventual consistency: namely, eventual consensus and eventual total order broadcast, and we prove them to be equivalent. We show in Section 4 that the weakest failure detector for eventual consensus in any message-passing environment is $\Omega$. We present in Section 5 our algorithm that implements eventual total order broadcast using $\Omega$ in any environment. Section 6 discusses related work, and Section 7 concludes the paper.

\section{PRELIMINARIES}

We adopt the classical model of distributed systems provided with the failure detector abstraction proposed in $[3,2]$. In particular we employ the simplified version of the model proposed in $[15,18]$.

We consider a message-passing system with a set of processes $\Pi=\left\{p_{1}, p_{2}, \ldots, p_{n}\right\}(n \geq 2)$. Processes execute steps of computation asynchronously, i.e., there is no bound on the delay between steps. However, we assume a discrete global clock to which the processes do not have access. The range of this clock's ticks is $\mathbb{N}$. Each pair of processes are connected by a reliable link.

Processes may fail by crashing. A failure pattern is a function $F: \mathbb{N} \rightarrow 2^{\Pi}$, where $F(t)$ is the set of processes that have crashed by time $t$. We assume that processes never recover from crashes, i. e., $F(t) \subseteq F(t+1)$. Let faulty $(F)=\bigcup_{t \in \mathbb{N}} F(t)$ be the set of faulty processes in a failure pattern $F$; and $\operatorname{correct}(F)=\Pi-\operatorname{faulty}(F)$ be the set of correct processes in $F$. An environment, denoted $\mathcal{E}$, is a set of failure patterns.

A failure detector history $H$ with range $\mathcal{R}$ is a function $H: \Pi \times \mathbb{N} \rightarrow \mathcal{R}$, where $H(p, t)$ is interpreted as the value output by the failure detector module of process $p$ at time $t$. A failure detector $\mathcal{D}$ with range $\mathcal{R}$ is a function that maps every failure pattern $F$ to a nonempty set of failure detector histories. $\mathcal{D}(F)$ denotes the set of all possible failure detector histories that may be output by $\mathcal{D}$ in a failure pattern $F$.

For example, at each process, the leader failure detector $\Omega$ outputs the id of a process; furthermore, if a correct process exists, then there is a time after which $\Omega$ outputs the id of the same correct process at every correct process. Another example is the quorum failure detector $\Sigma$, which outputs a set of processes at each process. Any two sets output at any times and by any processes intersect, and eventually every set output at any correct process consists of only correct processes.

An algorithm $\mathcal{A}$ is modeled as a collection of $n$ deterministic automata, where $\mathcal{A}(p)$ specifies the behavior of process $p$. Computation proceeds in steps of these automata. In each step, identified as a tuple $(p, m, d, \mathcal{A})$, a process $p$ atomically (1) receives a single message $m$ (that can be the empty message $\lambda$ ) or accepts an input (from the external world), (2) queries its local failure detector module and receives a value $d$, (3) changes its state according to $\mathcal{A}(p)$, and (4) sends a message specified by $\mathcal{A}(p)$ for the new state to every process or produces an output (to the external world). Note that the use of $\lambda$ ensures that a step of a process is always enabled, even if no message is sent to it.

A configuration of an algorithm $\mathcal{A}$ specifies the local state of each process and the set of messages in transit. In the initial configuration of $\mathcal{A}$, no message is in transit and each process $p$ is in the initial state of the automaton $\mathcal{A}(p)$. A 
schedule $S$ of $\mathcal{A}$ is a finite or infinite sequence of steps of $\mathcal{A}$ that respects $\mathcal{A}(p)$ for each $p$.

Following [18], we model inputs and outputs of processes using input histories $H_{I}$ and output histories $H_{O}$ that specify the inputs each process receives from its application and the outputs each process returns to the application over time. A run of algorithm $\mathcal{A}$ using failure detector $\mathcal{D}$ in environment $\mathcal{E}$ is a tuple $R=\left(F, H, H_{I}, H_{O}, S, T\right)$, where $F$ is a failure pattern in $\mathcal{E}, H$ is a failure detector history in $\mathcal{D}(F), H_{I}$ and $H_{O}$ are input and output histories of $\mathcal{A}, S$ is a schedule of $\mathcal{A}$, and $T$ is a list of increasing times in $\mathbb{N}$, where $T[i]$ is the time when step $S[i]$ is taken. $H \in \mathcal{D}(F)$, the failure detector values received by steps in $S$ are consistent with $H$, and $H_{I}$ and $H_{O}$ are consistent with $S$. An infinite run of $\mathcal{A}$ is admissible if (1) every correct process takes an infinite number of steps in $S$; and (2) each message sent to a correct process is eventually received.

We then define a distributed-computing problem, such as consensus or total order broadcast, as a set of tuples $\left(H_{I}, H_{O}\right)$ where $H_{I}$ is an input history and $H_{O}$ is an output history. An algorithm $\mathcal{A}$ using a failure detector $\mathcal{D}$ solves a problem $P$ in an environment $\mathcal{E}$ if in every admissible run of $\mathcal{A}$ in $\mathcal{E}$, the input and output histories are in $P$. Typically, inputs and outputs represent invocations and responses of operations exported by the implemented abstraction. If there is an algorithm that solves $P$ using $\mathcal{D}$, we sometimes, with a slight language abuse, say that $\mathcal{D}$ implements $P$.

Consider two problems $P$ and $P^{\prime}$. A transformation from $P$ to $P^{\prime}$ in an environment $\mathcal{E}[17]$ is a map $T_{P \rightarrow P^{\prime}}$ that, given any algorithm $\mathcal{A}_{P}$ solving $P$ in $\mathcal{E}$, yields an algorithm $\mathcal{A}_{P^{\prime}}$ solving $P^{\prime}$ in $\mathcal{E}$. The transformation is asynchronous in the sense that $\mathcal{A}_{P}$ is used as a "black box" where $\mathcal{A}_{P^{\prime}}$ is obtained by feeding inputs to $\mathcal{A}_{P}$ and using the returned outputs to solve $P^{\prime}$. Hence, if $P$ is solvable in $\mathcal{E}$ using a failure detector $\mathcal{D}$, the existence of a transformation $T_{P \rightarrow P^{\prime}}$ in $\mathcal{E}$ establishes that $P^{\prime}$ is also solvable in $\mathcal{E}$ using $\mathcal{D}$. If, additionally, there exists a transformation from $P^{\prime}$ to $P$ in $\mathcal{E}$, we say that $P$ and $P^{\prime}$ are equivalent in $\mathcal{E}$.

Failure detectors can be partially ordered based on their "power": failure detector $\mathcal{D}$ is weaker than failure detector $\mathcal{D}^{\prime}$ in $\mathcal{E}$ if there is an algorithm that emulates the output of $\mathcal{D}$ using $\mathcal{D}^{\prime}$ in $\mathcal{E}[2,18]$. If $\mathcal{D}$ is weaker than $\mathcal{D}^{\prime}$, any problem that can be solved with $\mathcal{D}$ can also be solved with $\mathcal{D}^{\prime}$. For a problem $P, \mathcal{D}^{*}$ is the weakest failure detector to solve $P$ in $\mathcal{E}$ if (a) there is an algorithm that uses $\mathcal{D}^{*}$ to solve $P$ in $\mathcal{E}$, and (b) $\mathcal{D}^{*}$ is weaker than any failure detector $\mathcal{D}$ that can be used to solve $P$ in $\mathcal{E}$.

\section{ABSTRACTIONS FOR EVENTUAL CONSISTENCY}

We define two basic abstractions that capture the notion of eventual consistency: eventual total order broadcast and eventual consensus. We show that the two abstractions are equivalent: each of them can be used to implement the other.

\section{Eventual Total Order Broadcast (ETOB).}

The total order broadcast (TOB) abstraction [17] exports one operation broadcastTOB $(m)$ and maintains, at every process $p_{i}$, an output variable $d_{i}$. Let $d_{i}(t)$ denote the value of $d_{i}$ at time $t$. Intuitively, $d_{i}(t)$ is the sequence of messages $p_{i}$ delivered by time $t$. We write $m \in d_{i}(t)$ if $m$ appears in $d_{i}(t)$.

A process $p_{i}$ broadcasts a message $m$ at time $t$ by a call to broadcastTOB $(m)$. We say that a process $p_{i}$ stably delivers a message $m$ at time $t$ if $p_{i}$ appends $m$ to $d_{i}(t)$ and $m$ is never removed from $d_{i}$ after that, i.e., $m \notin d_{i}(t-1)$ and $\forall t^{\prime} \geq t: m \in d_{i}\left(t^{\prime}\right)$. Note that if a message is delivered but not stably delivered by $p_{i}$ at time $t$, it appears in $d_{i}(t)$ but not in $d_{i}\left(t^{\prime}\right)$ for some $t^{\prime}>t$.

Assuming that broadcast messages are distinct, the TOB abstraction satisfies:

TOB-Validity If a correct process $p_{i}$ broadcasts a message $m$ at time $t$, then $p_{i}$ eventually stably delivers $m$, i.e., $\forall t^{\prime \prime} \geq t^{\prime}: m \in d_{i}\left(t^{\prime \prime}\right)$ for some $t^{\prime}>t$.

TOB-No-creation If $m \in d_{i}(t)$, then $m$ was broadcast by some process $p_{j}$ at some time $t^{\prime}<t$.

TOB-No-duplication No message appears more than once in $d_{i}(t)$.

TOB-Agreement If a message $m$ is stably delivered by some correct process $p_{i}$ at time $t$, then $m$ is eventually stably delivered by every correct process $p_{j}$.

TOB-Stability For any correct process $p_{i}, d_{i}\left(t_{1}\right)$ is a prefix of $d_{i}\left(t_{2}\right)$ for all $t_{1}, t_{2} \in \mathbb{N}, t_{1} \leq t_{2}$

TOB-Total-order Let $p_{i}$ and $p_{j}$ be any two correct processes such that two messages $m_{1}$ and $m_{2}$ appear in $d_{i}(t)$ and $d_{j}(t)$ at time $t$. If $m_{1}$ appears before $m_{2}$ in $d_{i}(t)$, then $m_{1}$ appears before $m_{2}$ in $d_{j}(t)$.

We then introduce the eventual total order broadcast (ETOB) abstraction, which maintains the same inputs and outputs as TOB (messages are broadcast by a call to broadcastETOB $(m)$ ) and satisfies, in every admissible run, the TOB-Validity, TOB-No-creation, TOB-No-duplication, and TOB-Agreement properties, plus the following relaxed properties for some $\tau \in \mathbb{N}$ :

ETOB-Stability For any correct process $p_{i}, d_{i}\left(t_{1}\right)$ is a prefix of $d_{i}\left(t_{2}\right)$ for all $t_{1}, t_{2} \in \mathbb{N}, \tau \leq t_{1} \leq t_{2}$.

ETOB-Total-order Let $p_{i}$ and $p_{j}$ be correct processes such that messages $m_{1}$ and $m_{2}$ appear in $d_{i}(t)$ and $d_{j}(t)$ for some $t \geq \tau$. If $m_{1}$ appears before $m_{2}$ in $d_{i}(t)$, then $m_{1}$ appears before $m_{2}$ in $d_{j}(t)$.

As we show in this paper, satisfying the following optional (but useful) property in ETOB does not require more information about failures.

TOB-Causal-Order Let $p_{i}$ be a correct process such that two messages $m_{1}$ and $m_{2}$ appear in $d_{i}(t)$ at time $t \in \mathbb{N}$. If $m_{2}$ depends causally of $m_{1}$, then $m_{1}$ appears before $m_{2}$ in $d_{i}(t)$.

Here we say that a message $m_{2}$ causally depends on a message $m_{1}$ in a run $R$, and write $m_{1} \rightarrow_{R} m_{2}$, if one of the following conditions holds in $R$ : (1) a process $p_{i}$ sends $m_{1}$ and then sends $m_{2},(2)$ a process $p_{i}$ receives $m_{1}$ and then sends $m_{2}$, or (3) there exists $m_{3}$ such that $m_{1} \rightarrow_{R} m_{3}$ and $m_{3} \rightarrow_{R} m_{2}$.

\section{Eventual Consensus (EC).}

The consensus abstraction (C) [12] exports, to every process $p_{i}$, a single operation propose $C$ that takes a binary argument and returns a binary response (we also say decides) so that the following properties are satisfied:

C-Termination Every correct process eventually returns a response to propose $C$.

C-Integrity Every process returns a response at most once. C-Agreement No two processes return different values. 
C-Validity Every value returned was previously proposed.

The eventual consensus (EC) abstraction exports, to every process $p_{i}$, operations propose $E C_{1}$, propose $E C_{2}, \ldots$ that take binary arguments and return binary responses. Assuming that, for all $j \in \mathbb{N}$, every process invokes propose $E C_{j}$ as soon as it returns a response to propose $E C_{j-1}$, the abstraction guarantees that, in every admissible run, there exists $k \in \mathbb{N}$, such that the following properties are satisfied:

EC-Termination Every correct process eventually returns a response to propose $E C_{j}$ for all $j \in \mathbb{N}$.

EC-Integrity No process responds twice to propose $E C_{j}$ for all $j \in \mathbb{N}$.

EC-Validity Every value returned to propose $E C_{j}$ was previously proposed to propose $E C_{j}$ for all $j \in \mathbb{N}$.

EC-Agreement No two processes return different values to propose $E C_{j}$ for all $j \geq k$.

It is straightforward to transform the binary version of EC into a multivalued one with unbounded set of inputs [24]. In the following, by referring to $\mathrm{EC}$ we mean a multivalued version of it.

\section{Equivalence between $\mathrm{EC}$ and $\mathrm{ETOB}$.}

It is well known that, in their classical forms, the consensus and the total order broadcast abstractions are equivalent [3]. In this section, we show that a similar result holds for our eventual versions of these abstractions.

The intuition behind the transformation from EC to ETOB is the following. Each time a process $p_{i}$ wants to ETOBbroadcast a message $m, p$ sends $m$ to each process. Periodically, every process $p_{i}$ proposes its current sequence of messages received so far to EC. This sequence is built by concatenating the last output of EC(stored in a local variable $d_{i}$ ) to the batch of all messages received by the process and not yet present in $d_{i}$. The output of EC is stored in $d_{i}$, i.e., at any time, each process delivers the last sequence of messages returned by EC.

The correctness of this transformation follows from the fact that ECeventually returns consistent responses to the processes. Thus, eventually, all processes agree on the same linearly growing sequence of stably delivered messages. Furthermore, every message broadcast by a correct process eventually appears either in the delivered message sequence or in the batches of not yet delivered messages at all correct processes. Thus, by EC-Validity of EC, every message ETOB-broadcast by a correct process is eventually stored in $d_{i}$ of every correct process $p_{i}$ forever. By construction, no message appears in $d_{i}$ twice or if it was not previously ETOB-broadcast. Therefore, the transformation satisfies the properties of ETOB.

The transformation from ETOB to EC is as follows. At each invocation of the $E C$ primitive, the process broadcasts a message using the ETOB abstraction. This message contains the proposed value and the index of the consensus instance. As soon as a message corresponding to a given eventual consensus instance is delivered by process $p_{i}$ (appears in $d_{i}$ ), $p_{i}$ returns the value contained in the message.

Since the ETOB abstraction guarantees that every process eventually stably delivers the same sequence of messages, there exists a consensus instance after which the responses of the transformation to all alive processes are identical. Moreover, by ETOB-Validity, every message ETOB-broadcast by a correct process $p_{i}$ is eventually stably delivered. Thus, every correct process eventually returns from any EC-instance it invokes. Thus, the transformation satisfies the EC specification.

THEOREM 1. In any environment $\mathcal{E}, \mathrm{EC}$ and $\mathrm{ETOB}$ are equivalent.

From EC to ETOB. To prove this result, it is sufficient to provide a protocol that implements ETOB in an environment $\mathcal{E}$ knowing that there exists a protocol that implements EC in this environment. This transformation protocol $\mathcal{T}_{\text {EC } \rightarrow \text { ETOB }}$ is stated in Algorithm 1. Now, we are going to prove that $\mathcal{T}_{\mathrm{EC} \rightarrow \mathrm{ETOB}}$ implements ETOB. Assume that there exists a message $m$ broadcast by a correct process $p_{i}$ at time $t$. As $p_{i}$ is correct, every correct process receives the message $\operatorname{push}(m)$ in a finite time. Then, $m$ appears in the set toDeliver of all correct processes in a finite time. Hence, by the termination property of EC and the construction of the function NewBatch, there exists $\ell$ such that $m$ is included in any sequence submitted to propose $E C_{\ell}$. By the EC-Validity and the EC-Termination properties, we deduce that $p_{i}$ stably delivers $m$ in a finite time, that proves that $\mathcal{T}_{\mathrm{EC} \rightarrow \mathrm{ETOB}}$ satisfies the TOB-Validity property. If a process $p_{i}$ delivers a message $m$ at time $t$, then $m$ appears in the sequence responded by its last invocation of proposeE $C_{\ell}$. By construction and by the EC-Validity property, this sequence contains only messages that appear in the set toDeliver of a process $p_{j}$ at the time $p_{j}$ invokes propose $E C_{\ell}$. But this set is incrementally built at the reception of messages push that contains only messages broadcast by a process. This implies that $\mathcal{T}_{\text {EC } \rightarrow \text { ETOB }}$ satisfies the TOB-No-creation. As the sequence outputted at any time by any process is the response to its last invocation of proposeEC and that the sequence submitted to any invocation of this primitive contains no duplicated message (by definition of the function NewBatch), we can deduce from the EC-Validity property that $\mathcal{T}_{\mathrm{EC} \rightarrow \mathrm{ETOB}}$ satisfies the TOB-No-duplication. Assume that a correct process $p_{i}$ stably delivers a message $m$, i.e., there exists a time after which $m$ always appears in $d_{i}$. By the algorithm, $m$ always appears in the response of proposeEC to $p_{i}$ after this time. As EC-Agreement property is eventually satisfied, we can deduce that $m$ always appears in the response of proposeEC for any correct process after some time. Thus, any correct process stably delivers $m$, and $\mathcal{T}_{\mathrm{EC} \rightarrow \text { ETOB }}$ satisfies the TOB-Agreement. Let $\tau$ be the time after which the EC primitive satisfies EC-Agreement and EC-Validity. Let $p_{i}$ be a correct process and $\tau \leq t_{1} \leq t_{2}$. Let $\ell_{1}$ (respectively $\ell_{2}$ ) be the integer such that $d_{i}\left(t_{1}\right)$ (respectively $d_{i}\left(t_{2}\right)$ ) is the response of propose $E C_{\ell_{1}}$ (respectively propose $E C_{\ell_{2}}$ ). By construction of the protocol and the EC-Agreement and EC-Validity properties, we know that, after time $\tau$, the response of propose $E C_{\ell}$ to correct processes is a prefix of the response of proposeE $C_{\ell+1}$. As we have $\ell_{1} \leq \ell_{2}$, we can deduce that $\mathcal{T}_{\mathrm{EC} \rightarrow \text { ETOB }}$ satisfies the ETOB-Stability property. Let $p_{i}$ and $p_{j}$ be two correct processes such that two messages $m_{1}$ and $m_{2}$ appear in $d_{i}(t)$ and $d_{j}(t)$ at time $t \geq \tau$. Let $\ell$ be the smallest integer such that $m_{1}$ and $m_{2}$ appear in the response of proposeE $C_{\ell}$. By the EC-Agreement property, we know that the response of propose $E C_{\ell}$ is identical for all correct processes. Then, by the ETOB-Stability property proved above, that implies that, if $m_{1}$ appears before $m_{2}$ in $d_{i}(t)$, then $m_{1}$ appears before $m_{2}$ in $d_{j}(t)$. In other words, $\mathcal{T}_{\text {EC } \rightarrow \text { ETOB }}$ satisfies the ETOB-Total-order property. In conclusion, $\mathcal{T}_{\mathrm{EC} \rightarrow \text { ETOB }}$ satisfies the ETOB specification in an environment $\mathcal{E}$ provided that there exists a protocol that implements EC in this environment. 


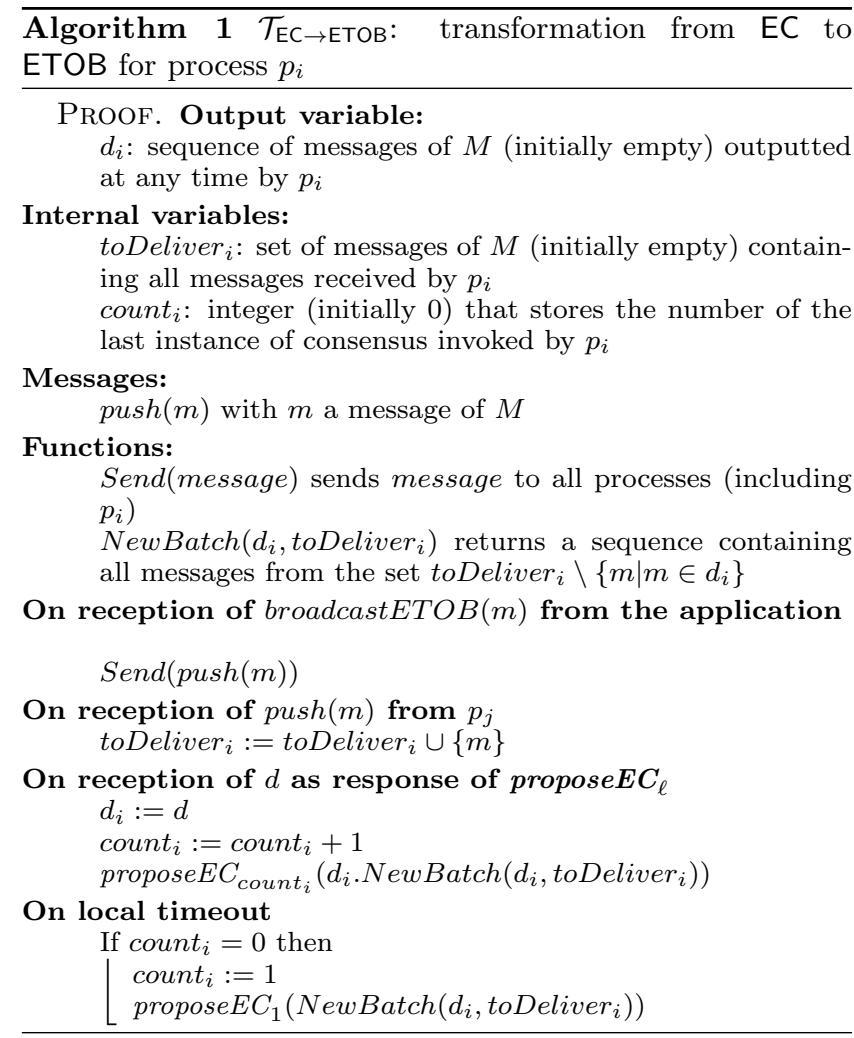

\section{From ETOB to EC.}

To prove this result, it is sufficient to provide a protocol that implements EC in an environment $\mathcal{E}$ given a protocol that implements ETOB in this environment. This transformation protocol $\mathcal{T}_{\mathrm{ETOB}} \rightarrow \mathrm{EC}$ is stated in Algorithm 2. Now, we are going to prove that $\mathcal{T}_{\mathrm{ETOB} \rightarrow \mathrm{EC}}$ implements $\mathrm{EC}$.

Let $p_{i}$ be a correct process that invokes proposeE $C_{\ell}(v)$ with $\ell \in \mathbb{N}$. Then, by fairness and the TOB-Validity property, the construction of the protocol implies that the ETOB primitive delivers the message $(\ell, v)$ to $p_{i}$ in a finite time. By the use of the local timeout, we know that $p_{i}$ returns from propose $E C_{\ell}(v)$ in a finite time, that proves that $\mathcal{T}_{\text {ETOB } \rightarrow \text { EC }}$ satisfies the EC-Termination property.

The update of the variable count $_{i}$ to $\ell$ for any process $p_{i}$ that invokes proposeE $C_{\ell}$ and the assumptions on operations proposeEC ensure us that $p_{i}$ executes at most once the function DecideEC $\left(\ell\right.$, received $\left._{i}\left[\Omega_{i}, \ell\right]\right) . \quad$ Hence, $\mathcal{T}_{\mathrm{ETOB} \rightarrow \mathrm{EC}}$ satisfies the EC-Integrity property.

Let $\tau$ be the time after which the ETOB-Stability and the ETOB-Total-order properties are satisfied. Let $k$ be the smallest integer such that any process that invokes propose $E C_{k}$ in run $r$ invokes it after $\tau$.

If we assume that there exist two correct processes $p_{i}$ and $p_{j}$ that return different values to propose $E C_{\ell}$ with $\ell \geq k$, we obtain a contradiction with the ETOB-Stability, ETOBTotal-order, or TOB-Agreement property. Indeed, if $p_{i}$ returns a value after time $\tau$, that implies that this value appears in $d_{i}$ and then, by the TOB-Agreement property, this value eventually appears in $d_{j}$. If $p_{j}$ returns a different value from $p_{i}$, that implies that this value is the first occurrence of a message associated to propose $E C_{\ell}$ in $d_{j}$ at the time of the return of propose $E C_{\ell}$. After that, $d_{j}$ cannot satisfy simultaneously the ETOB-Stability and the ETOB-Total- order properties. This contradiction shows that $\mathcal{T}_{\text {ETOB } \rightarrow \text { EC }}$ satisfies the EC-Agreement property.

If we assume that there exists a process $p_{i}$ that returns to propose $E C_{\ell}$ with $\ell \in \mathbb{N}$ a value that was not proposed to propose $E C_{\ell}$, we obtain a contradiction with the TOB-Nocreation property. Indeed, the return of $p_{i}$ from propose $E C_{\ell}$ is chosen in $d_{i}$ that contains the output of the ETOB primitive and processes broadcast only proposed values. This contradiction shows that $\mathcal{T}_{\mathrm{ETOB}} \rightarrow \mathrm{EC}$ satisfies the EC-Validity property.

In conclusion, $\mathcal{T}_{\mathrm{ETOB}} \rightarrow \mathrm{EC}$ satisfies the $\mathrm{EC}$ specification in an environment $\mathcal{E}$ provided that there exists a protocol that implements ETOB in this environment.

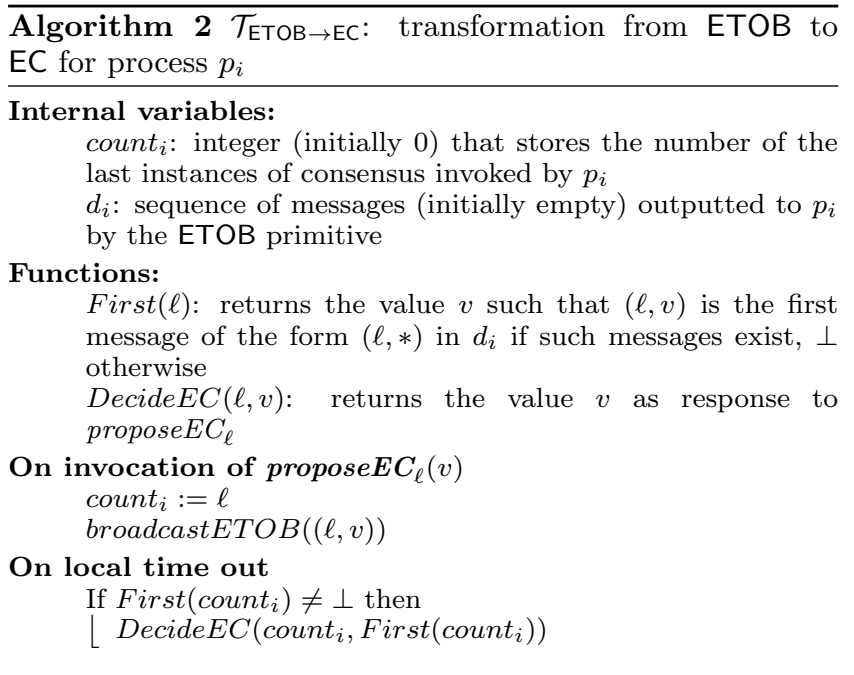

\section{THE WEAKEST FAILURE DETECTOR FOR EC}

In this section, we show that $\Omega$ is necessary and sufficient for implementing the eventual consensus abstraction EC:

THEOREM 2. In any environment $\mathcal{E}, \Omega$ is the weakest failure detector for $\mathrm{EC}$.

\section{$\Omega$ is necessary for $\mathrm{EC}$}

Let $\mathcal{E}$ be any environment. We show below that $\Omega$ is weaker than any failure detector $\mathcal{D}$ that can be used to solve $E C$ in $\mathcal{E}$. Recall that implementing $\Omega$ means outputting, at every process, the identifier of a leader process so that eventually, the same correct leader is output permanently at all correct processes.

First, we briefly recall the arguments use by Chandra et al. [2] in the original CHT proof deriving $\Omega$ from any algorithm solving consensus (to get a more detailed survey of the proof please rever to [13, Chapter 3]). The basic observation there is that a run of any algorithm using a failure detector induces a directed acyclic graph (DAG). The DAG contains a sample of failure detector values output by $\mathcal{D}$ in the current run and captures causal relations between them. Each process $p_{i}$ maintains a local copy of the DAG, denoted by $G_{i}$ : $p_{i}$ periodically queries its failure detector module, updates $G_{i}$ by connecting every vertex of the DAG with the vertex containing the returned failure-detector value with an edge, and broadcasts the DAG. An edge from vertex $\left[p_{i}, d, m\right]$ to 
vertex $\left[p_{j}, d^{\prime}, m^{\prime}\right]$ is thus interpreted as " $p_{i}$ queried $\mathcal{D}$ for the $m$ th time and obtained value $d$ and after that $p_{j}$ queried $\mathcal{D}$ for the $m^{\prime}$ th time and obtained value $d^{\prime \prime \prime}$. Whenever $p_{i}$ receives a DAG $G_{j}$ calculated earlier by $p_{j}, p_{i}$ merges $G_{i}$ with $G_{j}$. As a result, DAGs maintained by the correct processes converge to the same infinite DAG $G$. The DAG $G_{i}$ is then used by $p_{i}$ to simulate a number of runs of the given consensus algorithm $\mathcal{A}$ for all possible inputs to the processes. All these runs are organized in the form of a simulation tree $\Upsilon_{i}$. The simulation trees $\Upsilon_{i}$ maintained by the correct processes converge to the same infinite simulation tree $\Upsilon$.

The outputs produced in the simulated runs of $\Upsilon_{i}$ are then used by $p_{i}$ to compute the current estimate of $\Omega$. Every vertex $\sigma$ of $\Upsilon_{i}$ is assigned a valency tag based on the decisions taken in all its extensions (descendants of $\sigma$ in $\Upsilon_{i}$ ): $\sigma$ is assigned a tag $v \in\{0,1\}$ if $\sigma$ has an extension in which some process decides $v$. A vertex is bivalent if it is assigned both 0 and 1. It is then shown in [2] that by locating the same bivalent vertex in the limit tree $\Upsilon$, the correct process can eventually extract the identifier of the same correct process. (More details can be found in [2, 13].)

We show that this method, originally designed for consensus, can be extended to eventual consensus (i.e., to the weaker EC abstraction). The extension is not trivial and requires carefully adjusting the notion of valency of a vertex in the simulation tree.

LEMMA 3. In every environment $\mathcal{E}$, if a failure detector $\mathcal{D}$ implements $\mathrm{EC}$ in $\mathcal{E}$, then $\Omega$ is weaker than $\mathcal{D}$ in $\mathcal{E}$.

Proof. Let $\mathcal{A}$ be any algorithm that implements EC using a failure detector $\mathcal{D}$ in an environment $\mathcal{E}$. As in [2], every process $p_{i}$ maintains a failure detector sample stored in DAG $G_{i}$ and periodically uses $G_{i}$ to simulate a set of runs of $\mathcal{A}$ for all possible sequence of inputs of EC. The simulated runs are organized by $p_{i}$ in an ever-growing simulation tree $\Upsilon_{i}$. A vertex of $\Upsilon_{i}$ is the schedule of a finite run of $\mathcal{A}$ "triggered" by a path in $G_{i}$ in which every process starts with invoking propose $E C_{1}(v)$, for some $v \in\{0,1\}$, takes steps using the failure detector values stipulated by the path in $G_{i}$ and, once propose $E C_{\ell}(v)$ is complete, eventually invokes propose $E C_{\ell+1}\left(v^{\prime}\right)$, for some $v^{\prime} \in\{0,1\}$. (For the record, we equip each vertex of $\Upsilon_{i}$ with the path in $G_{i}$ used to produce it.) A vertex is connected by an edge to each one-step extension of it. ${ }^{2}$

Note that in every admissible infinite simulated run, ECTermination, EC-Integrity and EC-Validity are satisfied and that there is $k>0$ such that for all $\ell \geq k$, the invocations and responses of proposeE $C_{\ell}$ satisfy the EC-Agreement.

Since processes periodically broadcast their DAGs, the simulation tree $\Upsilon_{i}$ constructed locally by a correct process $p_{i}$ converges to an infinite simulation tree $\Upsilon$, in the sense that every finite subtree of $\Upsilon$ is eventually part of $\Upsilon_{i}$. The infinite simulation tree $\Upsilon$, starting from the initial configuration of $\mathcal{A}$ and, in the limit, contains all possible schedules that can triggered by the paths DAGs $G_{i}$.

Consider a vertex $\sigma$ in $\Upsilon$ identifying a unique finite schedule of a run of $\mathcal{A}$ using $\mathcal{D}$ in the current failure pattern $F$. For $k>0$, we say that $\sigma$ is $k$-enabled if $k=1$ or $\sigma$ contains a response from propose $E C_{k-1}$ at some process. Now

\footnotetext{
${ }^{2}$ In [2], the simulated schedules form a simulation forest, where a distinct simulation tree corresponds to each initial configuration encoding consensus inputs. Here we follow [18]: there is a single initial configuration and inputs are encoded in the form of input histories. As a result, we get a single simulation tree where branches depend on the parameters of propose $E C_{\ell}$ calls.
}

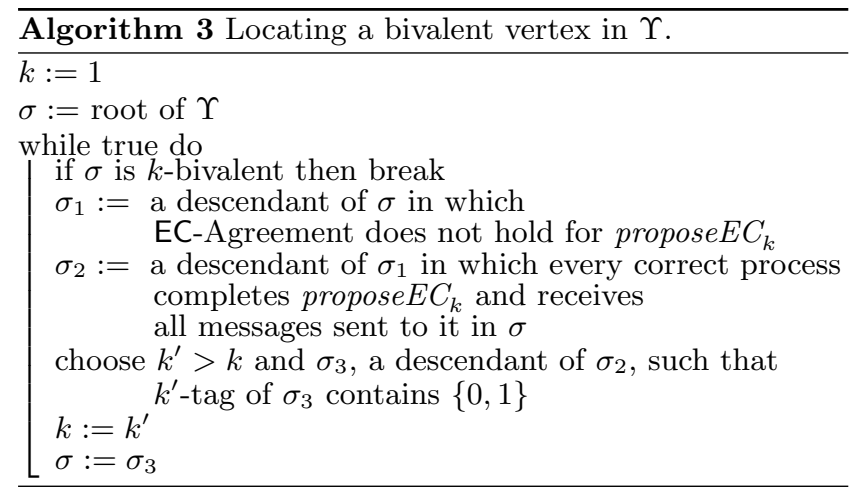

we associate each vertex $\sigma$ in $\Upsilon$ with a set of valency tags associated with each "consensus instance" $k$, called the $k$-tag of $\sigma$, as follows:

- If $\sigma$ is $k$-enabled and has a descendant (in $\Upsilon$ ) in which propose $E C_{k}$ returns $x \in\{0,1\}$, then $x$ is added to the $k$-tag of $\sigma$.

- If $\sigma$ is $k$-enabled and has a descendant in which two different values are returned by propose $E C_{k}$, then $\perp$ is added to the $k$-tag of $\sigma$.

If $\sigma$ is not $k$-enabled, then its $k$-tag is empty. If the $k$ tag of $\sigma$ is $\{x\}, x \in\{0,1\}$, we say that $\sigma$ is $(k, x)$-valent $(k$-univalent $)$. If the $k$-tag is $\{0,1\}$, then we say that $\sigma$ is $k$-bivalent. If the $k$-tag of $\sigma$ contains $\perp$, we say that $\sigma$ is $k$-invalid

Since $\mathcal{A}$ ensures EC-Termination in all admissible runs extending $\sigma$, each $k$-enabled vertex $\sigma$, the $k$-tag of $\sigma$ is nonempty. Moreover, EC-Termination and EC-Validity imply that a vertex in which no process has invoked proposeE $C_{k}$ yet has a descendant in which propose $E C_{k}$ returns 0 and a descendant in which propose $E C_{k}$ returns 1 . Indeed, a run in which only $v, v \in\{0,1\}$ is proposed in instance $k$ and every correct process takes enough steps must contain $v$ as an output. Thus:

$\left(^{*}\right)$ For each vertex $\sigma$, there exists $k \in \mathbb{N}$ and $\sigma^{\prime}$, a descendant of $\sigma$, such that $k$-tag of $\sigma^{\prime}$ contains $\{0,1\}$.

If the "limit tree" $\Upsilon$ contains a $k$-bivalent vertex, we can apply the arguments of [2] to extract $\Omega$. Now we show that such a vertex exists in $\Upsilon$. Then we can simply let every process locate the "first" such vertex in its local tree $\Upsilon_{i}$. To establish an order on the vertices, we can associate each vertex $\sigma$ of $\Upsilon$ with the value $m$ such that vertex $\left[p_{i}, d, m\right]$ of $G$ is used to simulate the last step of $\sigma$ (recall that we equip each vertex of $\Upsilon$ with the corresponding path). Then we order vertices of $\Upsilon$ in the order consistent with the growth of $m$. Since every vertex in $G$ has only finitely many incoming edges, the sets of vertices having the same value of $m$ are finite. Thus, we can break the ties in the $m$-based order using any deterministic procedure on these finite sets.

Eventually, by choosing the first $k$-bivalent vertex in their local trees $\Upsilon_{i}$, the correct processes will eventually stabilize on the same $k$-bivalent vertex $\tilde{\sigma}$ in the limit tree $\Upsilon$ and apply the CHT extraction procedure to derive the same correct process based on $k$-tags assigned to $\tilde{\sigma}$ 's descendants.

It remains to show that $\Upsilon$ indeed contains a $k$-bivalent vertex for some $k$. Consider the procedure described in Algorithm 3 that intends to locate such a vertex, starting with the root of the tree. 
For the currently considered $k$-enabled vertex $\sigma$ that is not $k$-bivalent (if it is $k$-bivalent, we are done), we use $\left(^{*}\right)$ to locate $\sigma_{3}$, a descendant of $\sigma$, such that (1) in $\sigma_{3}$, two processes return different values in proposeE $C_{k}$ in $\sigma_{3},(2)$ in $\sigma_{3}$, every correct process has completed propose $E C_{k}$ and has received every message sent to it in $\sigma$, and (3) the $k^{\prime}$-tag of $\sigma_{3}$ contains $\{0,1\}$.

Thus, the procedure in Algorithm 3 either terminates by locating a $k$-bivalent tag and then we are done, or it never terminates. Suppose, by contradiction, that the procedure never terminates. Hence, we have an infinite admissible run of $\mathcal{A}$ in which no agreement is provided in infinitely many instances of consensus. Indeed, in the constructed path along the tree, every correct process appears infinitely many times and receives every message sent to it. This admissible run violated the EC-Agreement property of EC - a contradiction.

Thus, the correct processes will eventually locate the same $k$-bivalent vertex and then, as in [2], stabilize extracting the same correct process identifier to emulate $\Omega$.

\section{$\Omega$ is sufficient for $\mathrm{EC}$.}

Chandra and Toueg proved that $\Omega$ is sufficient to implement the classical version of the consensus abstraction in an environment where a majority of processes are correct [3]. In this section, we extend this result to the eventual consensus abstraction for any environment.

The proposed implementation of EC is very simple. Each process has access to an $\Omega$ failure detector module. Upon each invocation of the EC primitive, a process broadcasts the proposed value (and the associated consensus index). Every process stores every received value. Each process $p_{i}$ periodically checks whether it has received a value for the current consensus instance from the process that it currently believes to be the leader. If so, $p_{i}$ returns this value. The correctness of this EC implementation relies on the fact that, eventually, all correct processes trust the same leader (by the definition of $\Omega$ ) and then decide (return responses) consistently on the values proposed by this process.

LEMMA 4. In every environment $\mathcal{E}$, EC can be implemented using $\Omega$.

Proof. We propose such an implementation in Algorithm 4. Then, we prove that any admissible run $r$ of the algorithm in any environment $\mathcal{E}$ satisfies the EC-Termination, EC-Integrity, EC-Agreement, and EC-Validity properties.

Assume that a correct process never returns from an invocation of proposeEC in $r$. Without loss of generality, denote by $\ell$ the smallest integer such that a correct process $p_{i}$ never returns from the invocation of propose $E C_{\ell}$. This implies that $p_{i}$ always evaluates received $d_{i}\left[\Omega_{i}\right.$, count $\left._{i}\right]$ to $\perp$. We know by definition of $\Omega$ that, eventually, $\Omega_{i}$ always returns the same correct process $p_{j}$. Hence, by construction of $\ell, p_{j}$ returns from propose $E C_{0}, \ldots$, propose $E C_{\ell-1}$ and then sends the message promote $(v, \ell)$ to all processes in a finite time. As $p_{i}$ and $p_{j}$ are correct, $p_{i}$ receives this message and updates received re $_{i}\left[\Omega_{i}\right.$, count $\left._{i}\right]$ to $v$ in a finite time. Therefore, the algorithm satisfies the EC-Termination property.

The update of the variable count $_{i}$ to $\ell$ for any process $p_{i}$ that invokes propose $E C_{\ell}$ and the assumptions on operations proposeEC ensure us that $p_{i}$ executes at most once the function

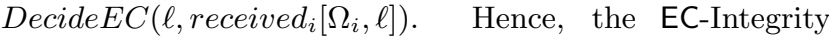
property is satisfied.

Let $\tau_{\Omega}$ be the time from which the local outputs of $\Omega$ are identical and constants for all correct processes in $r$. Let $k$ be the smallest integer such that any process that invokes proposeE $C_{k}$ in $r$ invokes it after $\tau_{\Omega}$.

Let $\ell$ be an integer such that $\ell \geq k$. Assume that $p_{i}$ and $p_{j}$ are two processes that respond to propose $E C_{\ell}$. Then, they respectively execute the function DecideEC $\left(\ell\right.$, received $\left._{i}\left[\Omega_{i}, \ell\right]\right)$ and DecideEC $\left._{\left(\ell, \text { received }_{j}\right.}\left[\Omega_{j}, \ell\right]\right)$. By construction of $k$, we can deduce that $\Omega_{i}=\Omega_{j}=p_{l}$. That implies that $p_{i}$ and $p_{j}$ both received a message promote $(v, \ell)$ from $p_{l}$. As $p_{l}$ sends such a message at most once, we can deduce that received $_{i}\left[p_{l}, \ell\right]=\operatorname{received}_{j}\left[p_{l}, \ell\right]$, that proves that ensures the EC-Agreement property.

Let $\ell$ be an integer such that $\ell \geq k$. Assume that $p_{i}$ is a process that respond to proposeEC $C_{\ell}$. The value returned by $p_{i}$ was previously received from $\Omega_{i}$ in a message of type promote. By construction of the protocol, $\Omega_{i}$ sends only one message of this type and this latter contains the value proposed to $\Omega_{i}$, hence, the EC-Validity property is satisfied.

Thus, Algorithm 4 indeed implements EC in any environment using $\Omega$.

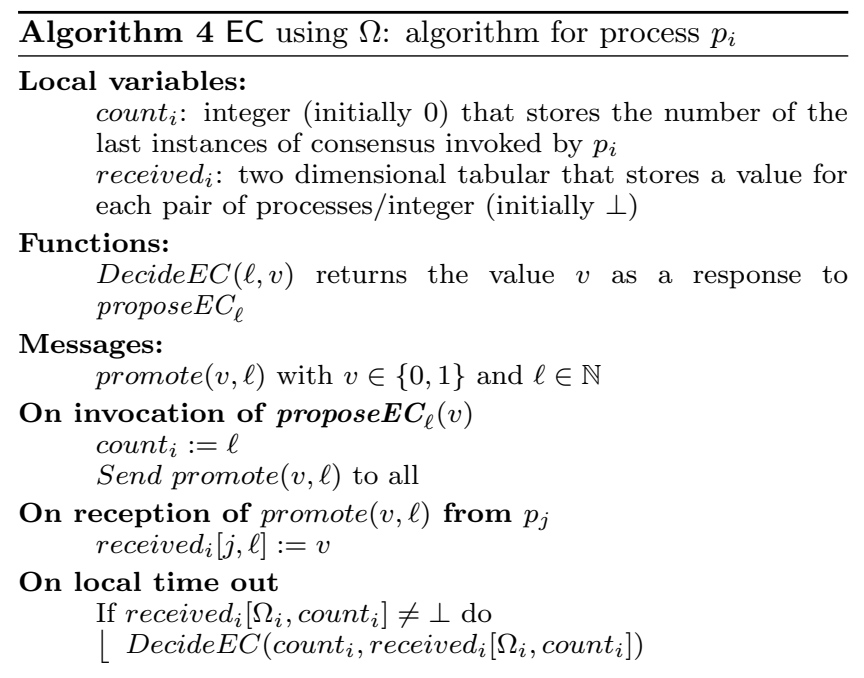

\section{AN EVENTUAL TOTAL ORDER BROADCAST ALGORITHM}

We have shown in the previous section that $\Omega$ is the weakest failure detector for the EC abstraction (and, by Theorem 1, the ETOB abstraction) in any environment. In this section, we describe an algorithm that directly implements ETOB using $\Omega$ and which we believe is interesting in its own right.

The algorithm has three interesting properties. First, it needs only two communication steps to deliver any message when the leader does not change, whereas algorithms implementing classical TOB need at least three communication steps in this case. Second, the algorithm actually implements total order broadcast if $\Omega$ outputs the same leader at all processes from the very beginning. Third, the algorithm additionally ensures the property of TOB-Causal-Order, which does not require more information about faults.

The intuition behind this algorithm is as follows. Every process that intends to ETOB-broadcast a message sends it to all other processes. Each process $p_{i}$ has access to an $\Omega$ failure detector module and maintains a DAG that stores the set of messages delivered so far together with their causal 


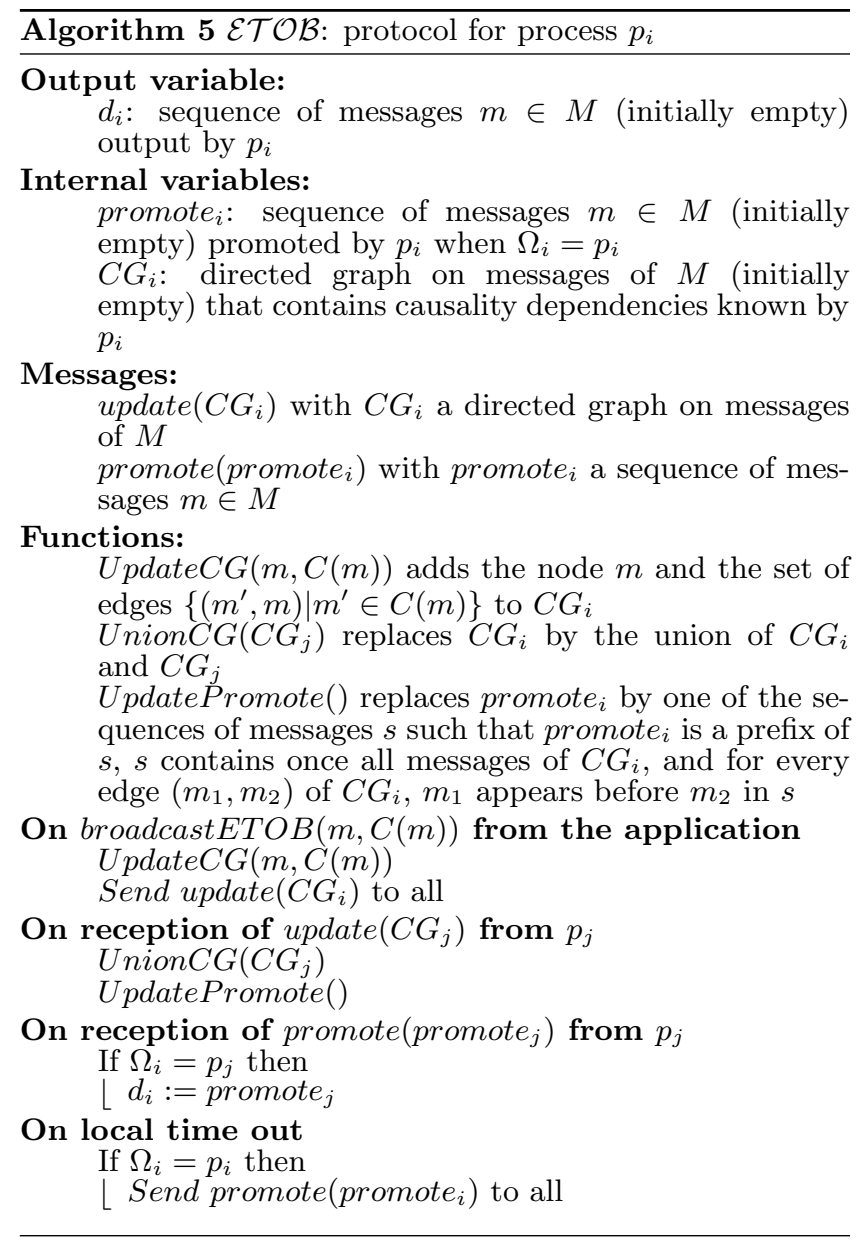

dependencies. As long as $p_{i}$ considers itself the leader (its module of $\Omega$ outputs $p_{i}$ ), it periodically sends to all processes a sequence of messages computed from its DAG so that the sequence respects the causal order and admits the last delivered sequence as a prefix. A process that receives a sequence of messages delivers it only if it has been sent by the current leader output by $\Omega$. The correctness of this algorithm directly follows from the properties of $\Omega$. Indeed, once all correct processes trust the same leader, this leader promotes its own sequence of messages, which ensures the ETOB specification.

The pseudocode of the algorithm is given in Algorithm 5). Below we present the proof of its correctness, including the proof that the algorithm additionally ensures TOB-CausalOrder.

LeMma 5. In every environment $\mathcal{E}$, Algorithm $\mathcal{E} \mathcal{T O B}$ implements $\mathrm{ETOB}$ using $\Omega$.

Proof. First, we prove that any run $r$ of $\mathcal{E} \mathcal{T O B}$ in any environment $\mathcal{E}$ satisfies the TOB-Validity, TOB-No-creation, TOB-No-duplication, and TOB-Agreement properties.

Assume that a correct process $p_{i}$ broadcasts a message $m$ at time $t$ for a given $t \in \mathbb{N}$. We know that $\Omega$ outputs the same correct process $p_{j}$ to all correct processes in a finite time. As $p_{j}$ is correct, it receives the message update $\left(C G_{i}\right)$ from $p_{i}$ (that contains $m$ ) in a finite time. Then, $p_{j}$ includes $m$ in its causality graph (by a call to Union $C G$ ) and in its promotion sequence (by a call to UpdatePromote). As $p_{j}$ never removes a message from its promotion sequence and is outputted by $\Omega, p_{i}$ adopts the promotion sequence of $p_{j}$ in a finite time and this sequence contains $m$, that proves that $\mathcal{E} \mathcal{T} \mathcal{O B}$ satisfies the TOB-Validity property.

Any sequence outputted by any process is built by a call to UpdatePromote by a process $p_{i}$. This function ensures that any message appearing in the computed sequence appears in the graph $C G_{p}$. This graph is built by successive calls to UnionCG that ensure that the graph contains only messages received in a message of type update. The construction of the protocol ensures us that such messages have been broadcast by a process. Then, we can deduce that $\mathcal{E} \mathcal{T O B}$ satisfies the TOB-No-creation property.

Any sequence outputted by any process is built by a call to UpdatePromote that ensures that any message appears only once. Then, we can deduce that $\mathcal{E} \mathcal{T} \mathcal{O B}$ satisfies the TOB-No-duplication property.

Assume that a correct process $p_{i}$ stably delivers a message $m$ at time $t$ for a given $t \in \mathbb{N}$. We know that $\Omega$ outputs the same correct process $p_{j}$ to all correct processes after some finite time. Since $m$ appears in every $d_{i}\left(t^{\prime}\right)$ such that $t^{\prime} \geq t$, we derive that $m$ appears infinitely in promote $_{j}$ from a given point of the run. Hence, the construction of the protocol and the correctness of $p_{j}$ implies that any correct process eventually stably delivers $m$, and $\mathcal{E} \mathcal{T O B}$ satisfies the TOBAgreement property.

We now prove that, for any environment $\mathcal{E}$, for any run $r$ of $\mathcal{E} \mathcal{T O B}$ in $\mathcal{E}$, there exists a $\tau \in \mathbb{N}$ satisfying ETOB-Stability, ETOB-Total-order, and TOB-Causal-Order properties in $r$. Hence, let $r$ be a run of $\mathcal{E} \mathcal{T} \mathcal{O B}$ in an environment $\mathcal{E}$. Let us define:

- $\tau_{\Omega}$ the time from which the local outputs of $\Omega$ are identical and constant for all correct processes in $r$;

- $\Delta_{c}$ the longest communication delay between two correct processes in $r$;

- $\Delta_{t}$ the longest local timeout for correct processes in $r$;

- $\tau=\tau_{\Omega}+\Delta_{t}+\Delta_{c}$

Let $p_{i}$ be a correct process and $p_{j}$ be the correct elected by $\Omega$ after $\tau_{\Omega}$. Let $t_{1}$ and $t_{2}$ be two integers such that $\tau \leq t_{1} \leq t_{2}$. As the output of $\Omega$ is stable after $\tau_{\Omega}$ and the choice of $\tau$ ensures us that $p_{i}$ receives at least one message of type promote from $p_{j}$, we can deduce from the construction of the protocol that there exists $t_{3} \leq t_{1}$ and $t_{4} \leq t_{2}$ such that $d_{i}\left(t_{1}\right)=$ promote $_{j}\left(t_{3}\right)$ and $d_{i}\left(t_{2}\right)=$ promote $_{j}\left(t_{4}\right)$. But the function $U$ pdatePromote used to build promote prosures $_{j}$ that promote $_{j}\left(t_{3}\right)$ is a prefix of promote $_{j}\left(t_{4}\right)$. Then, $\mathcal{E} \mathcal{T O B}$ satisfies the ETOB-Stability property after time $\tau$.

Let $p_{i}$ and $p_{j}$ be two correct processes such that two messages $m_{1}$ and $m_{2}$ appear in $d_{i}(t)$ and $d_{j}(t)$ at time $t \geq \tau$. Assume that $m_{1}$ appears before $m_{2}$ in $d_{i}(t)$. Let $p_{k}$ be the correct elected by $\Omega$ after $\tau_{\Omega}$. As the output of $\Omega$ is stable after $\tau_{\Omega}$ and the choice of $\tau$ ensures us that $p_{i}$ and $p_{j}$ receive at least one message of type promote from $p_{j}$, the construction of the protocol ensures us that we can consider $t_{1}$ and $t_{2}$ such that $d_{i}(t)=$ promote $_{k}\left(t_{1}\right)$ and $d_{j}(t)=$ promote $_{k}\left(t_{2}\right)$. The definition of the function UpdatePromote executed by $p_{k}$ allows us to deduce that either $d_{i}(t)$ is a prefix of $d_{j}(t)$ or $d_{j}(t)$ is a prefix of $d_{i}(t)$. In both cases, we obtain that $m_{1}$ appears before $m_{2}$ in $d_{j}(t)$, that proves that $\mathcal{E} \mathcal{T} \mathcal{O B}$ satisfies the ETOB-Total-order property after time $\tau$.

Let $p_{i}$ be a correct process such that two messages $m_{1}$ and $m_{2}$ appear in $d_{i}(t)$ at time $t \geq 0$. Assume that $m_{1} \in C\left(m_{2}\right)$ when $m_{2}$ is broadcast. Let $p_{j}$ be the process trusted by $\Omega_{i}$ at the time $p_{i}$ adopts the sequence $d_{i}(t)$. If $m_{2}$ appears in 
$d_{i}(t)$, that implies that the edge $\left(m_{1}, m_{2}\right)$ appears in $C G_{j}$ at the time $p_{j}$ executes $U p d a t e P r o m o t e ~\left(s i n c e ~ p_{j}\right.$ previously executed UnionCG that includes at least $m$ and the set of edges $\left\{\left(m^{\prime}, m\right) \mid m^{\prime} \in C(m)\right\}$ in $\left.C G_{j}\right)$. The construction of UpdatePromote ensures us that $m_{1}$ appears before $m_{2}$ in $d_{i}(t)$, that proves that $\mathcal{E} \mathcal{T} \mathcal{O B}$ satisfies the TOB-CausalOrder property.

In conclusion, $\mathcal{E} \mathcal{T O B}$ is an implementation of ETOB assuming that processes have access to the $\Omega$ failure detector in any environment.

\section{RELATED WORK}

Modern data service providers such as Amazon's Dynamo [7], Yahoo's PNUTs [6] or Google Bigtable distributed storage [4] are intended to offer highly available services. They consequently replicate those services over several server processes. In order to tolerate process failures as well as partitions, they consider eventual consistency [25, 29, 28].

The term eventual consensus was introduced in [19]. It refers to one instance of consensus which stabilizes at the end; not multiple instances as we consider in this paper. In [9], a self-stabilizing form of consensus was proposed: assuming a self-stabilizing implementation of $\diamond S$ (also described in the paper) and executing a sequence of consensus instances, validity and agreement are eventually ensured. Their consensus abstraction is close to ours but the authors focused on the shared-memory model and did not address the question of the weakest failure detector.

In [11], the intuition behind eventual consistency was captured through the concept of eventual serializability. Two kinds of operations were defined: (1) a "stable" operation of which response needs to be totally ordered after all operations preceding it and (2) "weak" operations of which responses might not reflect all their preceding operations. Our ETOB abstraction captures consistency with respect to the "weak" operations. (Our lower bound on the necessity of $\Omega$ naturally extends to the stronger definitions.)

Our perspective on eventual consistency is closely related to the notion of eventual linearizability discussed recently in [27] and [16]. It is shown in [27] that the weakest failure detector to boost eventually linearizable objects to linearizable ones is $\diamond P$. We are focusing primarily on the weakest failure detector to implement eventual consistency, so their result is orthogonal to ours.

In [16], eventual linearizability is compared against linearizability in the context of implementing specific objects in a shared-memory context. It turns out that an eventually linearizable implementation of a fetch-and-increment object is as hard to achieve as a linearizable one. Our ETOB construction can be seen as an eventually linearizable universal construction: given any sequential object type, ETOB provides an eventually linearizable concurrent implementation of it. Brought to the message-passing environment with a correct majority, our results complement [16]: we show that in this setting, an eventually consistent replicated service (eventually linearizable object with a sequential specification) requires exactly the same information about failures as a consistent (linearizable) one.

\section{CONCLUDING REMARKS}

This paper defined the abstraction of eventual total order broadcast and proved its equivalence to eventual consensus: two fundamental building blocks to implement a general replicated state machine that ensures eventual consistency. We proved that the weakest failure detector to implement these abstractions is $\Omega$, in any message-passing environment. We could hence determine the gap between building a general replicated state machine that ensures consistency in a message-passing system and one that ensures only eventual consistency. In terms of information about failures, this gap is precisely captured by failure detector $\Sigma[8]$. In terms of time complexity, the gap is exactly one message delay: an operation on the strongly consistent replicated must, in the worst case, incur three communication steps [23], while one build using our eventually total order broadcast protocol completes an operation in the optimal number of two communication steps.

Our ETOB abstraction captures a form of eventual consistency implemented in multiple replicated services $[7,6,4]$. In addition to eventual consistency guarantees, such systems sometimes produce indications when a prefix of operations on the replicated service is committed, i.e., is not subject to further changes. A prefix of operations can be committed, e.g., in sufficiently long periods of synchrony, when a majority of correct processes elect the same leader and all incoming and outgoing messages of the leader to the correct majority are delivered within some fixed bound. We believe that such indications could easily be implemented, during the stable periods, on top of ETOB. Naturally, our results imply that $\Omega$ is necessary for such systems too.

Our EC abstraction assumes eventual agreement, but requires integrity and validity to be always ensured. Other definitions of eventual consensus could be considered. In particular, we have studied an eventual consensus abstraction assuming, instead of eventual agrement, eventual integrity, i.e., a bounded number of decisions in a given consensus instance could be revoked a finite number of times. In the companion technical report [10], we define this abstraction of eventual irrevocable consensus (EIC) more precisely and show that it is equivalent to our EC abstraction.

\section{REFERENCES}

[1] E. A. Brewer. Towards robust distributed systems (abstract). In Proceedings of the Nineteenth Annual ACM Symposium on Principles of Distributed Computing, PODC '00, pages 7-, 2000.

[2] T. D. Chandra, V. Hadzilacos, and S. Toueg. The weakest failure detector for solving consensus. J. ACM, 43(4):685-722, July 1996.

[3] T. D. Chandra and S. Toueg. Unreliable failure detectors for reliable distributed systems. J. $A C M$, 43(2):225-267, Mar. 1996.

[4] F. Chang, J. Dean, S. Ghemawat, W. C. Hsieh, D. A. Wallach, M. Burrows, T. Chandra, A. Fikes, and R. E. Gruber. Bigtable: A distributed storage system for structured data. ACM Trans. Comput. Syst., 26(2):4:1-4:26, June 2008.

[5] B. Charron-Bost and G. Tel. Approximation d'une borne inférieure répartie. Technical Report LIX/RR/94/06, Laboratoire d'Informatique LIX, École Polytechnique, Sept. 1994.

[6] B. F. Cooper, R. Ramakrishnan, U. Srivastava, A. Silberstein, P. Bohannon, H.-A. Jacobsen, N. Puz, D. Weaver, and R. Yerneni. Pnuts: Yahoo!'s hosted data serving platform. Proc. VLDB Endow., 1(2):1277-1288, Aug. 2008.

[7] G. DeCandia, D. Hastorun, M. Jampani, G. Kakulapati, A. Lakshman, A. Pilchin, S. Sivasubramanian, P. Vosshall, and W. Vogels. Dynamo: Amazon's highly available key-value store. 
In Proceedings of Twenty-first ACM SIGOPS Symposium on Operating Systems Principles, SOSP '07, pages 205-220, New York, NY, USA, 2007. ACM.

[8] C. Delporte-Gallet, H. Fauconnier, and R. Guerraoui. Tight failure detection bounds on atomic object implementations. J. ACM, 57(4), 2010.

[9] S. Dolev, R. I. Kat, and E. M. Schiller. When consensus meets self-stabilization. Journal of Computer and System Sciences, 76(8):884 - 900, 2010.

[10] S. Dubois, R. Guerraoui, P. Kuznetsov, F. Petit, and P. Sens. The weakest failure detector for eventual consistency. CoRR, abs/X.X, 2015.

[11] A. Fekete, D. Gupta, V. Luchangco, N. Lynch, and A. Shvartsman. Eventually-serializable data services. In Proceedings of the Fifteenth Annual ACM Symposium on Principles of Distributed Computing, PODC '96, pages 300-309, New York, NY, USA, 1996. ACM.

[12] M. J. Fischer, N. A. Lynch, and M. S. Paterson. Impossibility of distributed consensus with one faulty process. J. ACM, 32(2):374-382, Apr. 1985.

[13] F. C. Freiling, R. Guerraoui, and P. Kuznetsov. The failure detector abstraction. ACM Comput. Surv., 43(2):9:1-9:40, Feb. 2011.

[14] S. Gilbert and N. Lynch. Brewer's conjecture and the feasibility of consistent, available, partition-tolerant web services. SIGACT News, 33(2):51-59, June 2002.

[15] R. Guerraoui, V. Hadzilacos, P. Kuznetsov, and S. Toueg. The weakest failure detectors to solve quittable consensus and nonblocking atomic commit. SIAM J. Comput., 41(6):1343-1379, 2012.

[16] R. Guerraoui and E. Ruppert. A paradox of eventual linearizability in shared memory. In Proceedings of the 2014 ACM Symposium on Principles of Distributed Computing, PODC '14, pages 40-49, 2014.

[17] V. Hadzilacos and S. Toueg. A modular approach to fault-tolerant broadcasts and related problems. Technical Report TR 94-1425, Department of Computer Science, Cornell University, May 1994.

[18] P. Jayanti and S. Toueg. Every problem has a weakest failure detector. In $P O D C$, pages 75-84, 2008.
[19] F. Kuhn, Y. Moses, and R. Oshman. Coordinated consensus in dynamic networks. In Proceedings of the 30th Annual ACM Symposium on Principles of Distributed Computing (PODC), pages 1-10. ACM, 2011.

[20] A. Lakshman and P. Malik. Cassandra: A decentralized structured storage system. SIGOPS Oper. Syst. Rev., 44(2):35-40, Apr. 2010.

[21] L. Lamport. Proving the correctness of multiprocessor programs. Transactions on software engineering, 3(2):125-143, Mar. 1977.

[22] L. Lamport. The Part-Time parliament. ACM Transactions on Computer Systems, 16(2):133-169, May 1998.

[23] L. Lamport. Lower bounds for asynchronous consensus. Distributed Computing, 19(2):104-125, 2006.

[24] A. Mostefaoui, M. Raynal, and F. Tronel. From binary consensus to multivalued consensus in asynchronous message-passing systems. Inf. Process. Lett., 73(5-6):207-212, Mar. 2000.

[25] Y. Saito and M. Shapiro. Optimistic replication. ACM Comput. Surv., 37(1):42-81, Mar. 2005.

[26] F. B. Schneider. Implementing fault-tolerant services using the state machine approach: A tutorial. ACM Computing Surveys, 22(4):299-319, Dec. 1990.

[27] M. Serafini, D. Dobre, M. Majuntke, P. Bokor, and N. Suri. Eventually linearizable shared objects. In A. W. Richa and R. Guerraoui, editors, Proceedings of the 29th Annual ACM Symposium on Principles of Distributed Computing, pages 95-104. ACM, 2010.

[28] A. Singh, P. Fonseca, P. Kuznetsov, R. Rodrigues, and P. Maniatis. Zeno: Eventually consistent byzantine-fault tolerance. In Proceedings of the 6th USENIX Symposium on Networked Systems Design and Implementation, NSDI'09, pages 169-184, Berkeley, CA, USA, 2009. USENIX Association.

[29] W. Vogels. Eventually consistent. Commun. ACM, 52(1):40-44, Jan. 2009. 